\title{
Inhaltsverzeichnis
}

Verzeichnis der Bearbeiter der 3. Auflage - XIV

Abkürzungsverzeichnis $-\mathrm{XV}$

Verzeichnis der abgekürzt zitierten Literatur — XXVII

\section{Kapitel 2}

Rechtsfolgen -1

\section{§ 8 Beseitigung und Unterlassung - 1}

Schrifttum zu $\S 8$ bis $\S 8 b-1$

A. Einführung - 11

I. Entstehungsgeschichte -14

II. Inhalt und Zweck der Regelung - 17

III. Anwendungsbereich -19

B. Wettbewerbsrechtliche Abwehransprüche (Absatz 1) - 21

I. Systematik -21

II. Gesetzlicher Unterlassungsanspruch (Abs. 1 Satz 1 Alt. 2 und Satz 2) 21

III. Vertraglicher Unterlassungsanspruch -73

IV. Beseitigungsanspruch (Absatz 1 Satz 1 Alt. 1) -79

C. Schuldner der Abwehransprüche -90

I. Übersicht -90

II. Täter -92

III. Teilnehmer (Anstifter und Gehilfen) -121

IV. Haftung für Mitarbeiter und Beauftragte (Absatz 2) -123

V. Organ- und Repräsentantenhaftung - 134

VI. Haftung für Erfüllungs- und Verrichtungsgehilfen - 137

VII. Mehrheit von Schuldnern - 138

VIII. Rechtsnachfolge - 139

D. Gläubiger der Abwehransprüche (Absatz 3) - 141

I. Allgemeines - 141

II. Mitbewerber ( $\S 8$ Abs. 3 Nr. 1) -151

III. Wirtschaftsverbände (§ 8 Abs. 3 Nr. 2) -154

IV. Verbraucherverbände ( 88 Abs. 3 Nr. 3) -160

V. Berufsständische Körperschaften und Gewerkschaften (§ 8 Abs. 3 Nr. 4) -168

E. Spezieller Auskunftsanspruch (Absatz 5) - 169

I. Allgemeines -169

II. Wortlaut des $\S 13$ UKlaG -170

§ 8a Anspruchsberechtigte bei einem Verstoß gegen die Verordnung (EU) $2019 / 1150-171$

A. Einführung -171

B. Aktivlegitimation von Verbänden und öffentlichen Stellen nach

P2B-Verordnung - 172

\section{§ 8b Liste der qualifizierten Wirtschaftsverbände - 174}

A. Einführung -174

B. Qualifizierte Wirtschaftsverbände -175

I. Liste der qualifizierten Wirtschaftsverbände des Bundesamts für Justiz

(Abs. 1) -175 
II. Eintragungsvoraussetzungen (Abs. 2) -175

III. Anwendung bestimmter Normen des UKlaG (Abs. 3) -182

§ 8c Verbot der missbräuchlichen Geltendmachung von Ansprüchen; Haftung — 185

Schrifttum - 185

A. Einführung - 186

I. Vor- und Nachteile außergerichtlicher Rechtsdurchsetzung über „Abmahnungen“- 187

II. „Stellschrauben“ zur Begrenzung von Missbrauch — 187

III. Normzweck des Missbrauchstatbestandes - 188

IV. Entwicklung - 189

B. Missbräuchliche Geltendmachung von Abwehransprüchen — 190

I. Anwendungsbereich -190

II. Rechtsnatur - 191

III. Rechtsfolgen des Missbrauchs - 192

IV. Fallgruppen - 193

V. Gegenansprüche - 201

C. Prozessuales und Beweislast -202

D. Sonstige Einwände gegen die Rechtsdurchsetzung — 202

I. Materiell-rechtliche Einwendungen im engeren und im weiteren Sinne -202

II. Verwirkung - 203

$\S 9$ Schadensersatz -209

Schrifttum -209

A. Einführung -210

I. Entstehungsgeschichte -212

II. Bedeutung und Funktionen - 213

III. Unionsrechtliche Vorgaben und Einflüsse — 215

IV. Rechtsnatur und systematische Verortung - 217

V. Konkurrenzen - 217

B. Schadensersatzanspruch (Satz 1) - 221

I. Tatbestandsvoraussetzungen -221

II. Gläubiger und Schuldner - 229

III. Inhalt und Umfang - 236

IV. Dreifache Schadensberechnung - 249

Schrifttum - 249

V. Mitverschulden -270

C. Presseprivileg (Satz 2) -271

Schrifttum -271

I. Normzweck und Rechtsentwicklung - 272

II. Anwendungsbereich und Reichweite - 272

III. Darlegungs- und Beweislast -273

D. Bereicherungsanspruch -274

Schrifttum — 274

I. Anwendungsbereich und Grundsätzliches - 274

II. Voraussetzungen -275

III. Inhalt und Umfang - 278

IV. Haftung mehrerer und Mitverschulden - 282

V. Konkurrenzen und Verjährung - 282

E. Anspruch aus Geschäftsführung ohne Auftrag — 282 
F. Ansprüche auf Auskunft, Rechnungslegung und Besichtigung - 283

Schrifttum - 283

I. Grundsätzliches - 284

II. Tatbestand - 286

III. Inhalt und Umfang der Auskunfts- und Rechnungslegungspflicht -300

IV. Durchsetzung - 307

V. Verjährung -307

VI. Anspruch auf Besichtigung (§ 809 BGB) -308

\section{$\S 10$ Gewinnabschöpfung - 310}

\section{Schrifttum - 310}

Alphabetisches Stichwortverzeichnis - 316

A. Grundlagen - 317

I. Entstehungsgeschichte -317

II. Kollektive Rechtsdurchsetzung bei Streuschäden - 321

III. Zweck der Regelung - 335

IV. Rechtspolitische Diskussion - 338

V. Rechtsvergleichender Überblick - 349

VI. Rechtsnatur des Gewinnabschöpfungsanspruchs - 353

B. Anspruchsvoraussetzungen - 359

I. Vorsätzliches Fehlverhalten - 359

II. Gewinnerzielung zu Lasten einer Vielzahl von Abnehmern — 363

C. Rechtsfolgen - 375

I. Passivlegitimation $\mathbf{3} \mathbf{3 7 5}$

II. Aktivlegitimation -377

III. Abschöpfung von Gewinnen zugunsten des Bundeshaushalts — 381

IV. Anrechnung anderer Leistungen - 384

D. Durchsetzung und Verfahren - 389

I. Zulässigkeit der Klage - 389

II. Beweis - 396

III. Wirkung des Urteils -405

IV. Follow-on-Klagen -405

V. Verjährung - 406

E. Internationalrechtliche Fragen -407

I. Internationales Verfahrensrecht -407

II. Internationales Privatrecht -408

III. Anerkennung und Vollstreckbarkeit ausländischer Urteile -409

$\S 11$ Verjährung -410

\section{Schrifttum -410}

A. Einführung -411

I. Entstehungsgeschichte -412

II. Zweck der Verjährung - 414

III. Bedeutung und Systematik der Vorschrift -415

IV. Anwendungsbereich -417

B. Einzelheiten -430

I. Regelmäßige Verjährung (Absätze 1, 2) -430

II. Verjährungshöchstfristen (Absätze 3, 4) - 442

III. Einwirkung auf den Lauf der Verjährung — 442

IV. Rechtsfolgen der Verjährung — 453 
C. Verfahrensrechtliches -455

I. Prozessuale Behandlung der Verjährung - 455

II. Darlegungs- und Beweislast -457

\section{Kapitel 3}

Verfahrensvorschriften -459

Vorbemerkungen zu §§ 12-15a -459

A. Besonderheiten des Klageverfahrens - 459

I. Gerichtsbarkeit und Rechtsweg -459

Schrifttum - 459

II. Insbesondere: Das Rechtsschutzbedürfnis für die Unterlassungsklage -477 Schrifttum - 477

III. Unterlassungsklage -489

Schrifttum - 489

IV. Anderweitige Rechtshängigkeit und Klageänderung - 596

Schrifttum - 596

V. Besondere Klagearten -616

Schrifttum - 616

VI. Beweis und Beweislast -641

Schrifttum - 641

VII. Verfahrensunterbrechungen -667

Schrifttum -667

VIII. Rechtskraft -677

Schrifttum - 677

IX. Erledigung der Hauptsache -708

Schrifttum -708

X. Kosten -723

Schrifttum - 723

XI. Vergleich -738

Schrifttum - 738

XII. Zwangsvollstreckung - 755

Schrifttum -755

B. Aufbrauchfrist -820

Schrifttum -820

I. Einführung -820

II. Rechtsgrundlage -822

III. Voraussetzungen -828

IV. Rechtsfolgen -831

V. Dauer - 831

VI. Prozessuale Behandlung -832

§ 12 Einstweiliger Rechtsschutz, Veröffentlichungsbefugnis, Streitwertminderung — 836

Schrifttum - 836

A. Einstweilige Verfügung, Abschlusserklärung und Abschlussschreiben - 841

I. Einleitung - 846

II. Zuständigkeit -847

III. Inadäquater Verfügungsanspruch -852

IV. Verfügungsgrund -858

V. Das Gerichtsverfahren - 894 
VI. Anordnung der Klageerhebung (§ 926 ZPO) -939

VII. Der Aufhebungsantrag ( $\$ 927$ ZPO) -949

VIII. Das Abschlussschreiben - 960

IX. Die Abschlusserklärung - 969

$X$. Die Vollziehung der einstweiligen Verfügung - 976

XI. Schadensersatz nach § 945 ZPO — 997

XII. Die Zwangsvollstreckung aus der einstweiligen Verfügung - 1012

XIII. Die Rechtskraft der einstweiligen Verfügung — 1016

B. Urteilsveröffentlichung ( $§ 12$ Abs. 2) -1019

Schrifttum 1019

Alphabetisches Stichwortverzeichnis - 1019

I. Einleitung - 1020

II. Tatbestandsvoraussetzungen -1027

III. Art und Umfang der Bekanntmachung — 1033

IV. Veröffentlichungsfrist 1036

V. Kosten und Vollstreckung - 1037

C. Streitwert und Streitwertbegünstigung, § 12 Abs. 3 u. $4-1038$

Schrifttum -1038

I. Der volle Streitwert 1039

II. Streitwertbegünstigung - 1066

$\S 13$ Abmahnung; Unterlassungsverpflichtung; Haftung — 1076

Schrifttum -1076

Alphabetisches Stichwortverzeichnis - 1084

I. Abmahnung - 1088

II. Unterwerfung - 1153

$\S 13 a$ Vertragsstrafe -1203

Schrifttum - 1203

Alphabetisches Stichwortverzeichnis - 1205

I. Allgemeines - 1206

II. Verwirkung der Vertragsstrafe -1209

III. Höhe der Vertragsstrafe - 1216

IV. Ausschluss der Vertragsstrafe (§ 13a Abs. 2) - 1221

V. Rechtsmissbrauch - 1221

VI. Verjährung - 1223

VII. Verhältnis zu weiteren Sanktionen - 1224

§ 14 Sachliche und örtliche Zuständigkeit; Verordnungsermächtigung — 1227

A. Sachliche Zuständigkeit, Abs. $1-1227$

Schrifttum — 1228

Alphabetisches Stichwortverzeichnis - 1229

I. Entstehungsgeschichte -1230

II. Inhalt und Zweck der Regelung — 1234

III. Anwendungsbereich - 1236

IV. Prüfung der Zuständigkeitsvoraussetzungen — 1244

V. Besondere Verfahrensarten - 1246

VI. Zuständigkeit der Kammern für Handelssachen, § 13 Abs. 1 S. 2 a. F. -1249 
B. Örtliche Zuständigkeit, Abs. $2-1257$

Schrifttum - 1257

Alphabetisches Stichwortverzeichnis - 1259

I. Entstehungsgeschichte - 1260

II. Inhalt und Zweck der Regelung - 1275

III. Verfahrensfragen -1277

IV. Klagen auf Grund dieses Gesetzes - 1288

V. Allgemeiner Gerichtsstand des Beklagten, § 14 Abs. 2 S. $1-1288$

VI. Gerichtsstand der Niederlassung - 1291

VII. Gerichtsstand des Begehungsorts, § 14 Abs. 2 S. $2-1295$

VIII. Beschränkungen des Gerichtsstands des Begehungsorts -1317

C. Konzentrationsermächtigung, Abs. $3-1323$

$\S 15$ Einigungsstellen -1325

Schrifttum - 1326

Alphabetisches Stichwortverzeichnis - 1329

A. Einführung - 1331

I. $\quad$ Entstehungsgeschichte -1331

II. Inhalt und Zweck der Regelung - 1332

III. Anwendungsbereich -1333

B. Allgemeines zu Einigungsstellen - 1334

I. Historische Entwicklung - 1334

II. Rechtsquellen - 1340

III. Status und Wesen der Einigungsstellen - 1343

IV. Abgrenzung der Einigungsstellen — 1344

V. Vorzüge und Nachteile der Einigungsstellen, Kritik - 1348

C. Errichtung und Geschäftsführung, Aufsicht und Amtshaftung — 1353

I. Errichtung der Einigungsstellen — 1353

II. Geschäftsführung der Einigungsstellen - 1354

III. Aufsicht über die Einigungsstellen - 1355

IV. Amtshaftung bei Amtspflichtverletzungen - 1356

D. Besetzung der Einigungsstellen - 1357

I. Berufung zu Mitgliedern der Einigungsstelle - 1357

II. Besetzung der Einigungsstelle im konkreten Streitfall — 1365

III. Ausschließung und Ablehnung von Mitgliedern der Einigungsstelle - 1366

E. Zuständigkeit der Einigungsstelle - 1370

I. Örtliche Zuständigkeit - 1370

II. Sachliche Zuständigkeit - 1373

F. Verfahren der Einigungsstelle -1383

I. Antragsbefugnis - 1383

II. Antragserfordernis - 1384

III. Vorprüfung - 1386

IV. Vorbereitung der mündlichen Verhandlung — 1388

V. Mündliche Verhandlung - 1396

VI. Beendigung des Einigungsstellenverfahrens — 1414

VII. Kosten des Einigungsstellenverfahrens - 1423

G. Zwangsvollstreckung aus Einigungsstellenvergleichen - 1436

I. Allgemeine Voraussetzungen der Zwangsvollstreckung - 1436

II. Besondere Voraussetzungen der Zwangsvollstreckung - 1437

H. Materiellrechtliche Wirkungen des Einigungsstellenverfahrens - 1441

I. Keine Auswirkungen auf den geltend gemachten Anspruch — 1441 
II. Hemmung der Verjährung — 1441

III. Begründung eines Schuldverhältnisses — 1448

J. Verfahrensrechtliche Wirkungen des Einigungsstellenverfahrens - 1449

I. Anrufung der Einigungsstelle vor Einleitung gerichtlicher Verfahren - 1449

II. Anrufung der Einigungsstelle nach Einleitung gerichtlicher Verfahren -1457

\section{Anhang Durchführungsverordnungen der Länder — 1462}

§ 15a Überleitungsvorschrift zum Gesetz zur Stärkung des fairen Wettbewerbs — 1511

A. Übergangsregelung Aktivlegitimation Wirtschaftsverbände (Abs. 1) -1511

B. Überleitungsvorschrift für Abmahnung und Vertragsstrafe (§ 15a Abs. 2) - 1512

\section{Kapitel 4}

Straf- und Bußgeldvorschriften — 1513

\section{$\S 16$ Strafbare Werbung - 1513}

Schrifttum - 1513

Zum Lauterkeitsstrafrecht im Allgemeinen und zu Abs. $1-1513$

Zu Abs. $2-1515$

Alphabetisches Stichwortverzeichnis — 1516

A. Allgemeines 1517

I. Überblick zu den Straf- und Bußgeldvorschriften des UWG -1517

II. Entstehungsgeschichte 1518

III. Deliktsstruktur — 1519

IV. Strafverfolgungsbehörden und Gerichte - 1520

V. Praktische Bedeutung - 1520

B. Strafbare irreführende Werbung nach $\S 16$ Abs. $1-1521$

I. Vergleich zu \& 4 a. F. -1521

II. Schutzzweck der Norm - 1521

III. Verhältnis zu §§ 5, 5a- 1521

IV. Verhältnis zu $§ 263$ StGB 1522

V. Bestimmtheitsgebot (Art. 103 Abs. 2 GG) -1523

VI. Objektiver Tatbestand -1523

VII. Subjektiver Tatbestand - 1532

VIII. Rechtswidrigkeit und Schuld - 1536

IX. Vollendung und Beendigung - 1537

X. Täterschaft und Teilnahme -1538

XI. Verjährung - 1539

XII. Strafe und weitere Rechtsfolgen -1540

XIII. Konkurrenzen - 1542

XIV. Zivilrechtliche Folgen -1543

XV. Strafverfolgung - 1543

C. Strafbare progressive Kundenwerbung nach § 16 Abs. $2-1543$

I. Schutzzweck der Norm - 1543

II. Erscheinungsformen -1544

III. Strafwürdigkeit von Schneeball- und Pyramidensystemen - 1548

IV. Objektiver Tatbestand - 1549

V. Subjektiver Tatbestand - 1555

VI. Rechtswidrigkeit und Schuld -1556

VII. Täterschaft und Teilnahme - 1556 
VIII. Verjährung - 1557

IX. Strafe und weitere Rechtsfolgen - 1557

X. Konkurrenzen -1557

XI. Zivilrechtliche Folgen -1558

XII. Strafverfolgung - 1558

§§ 17-19 (weggefallen, ersetzt durch § 23 GeschGehG, s. S. 1578) - 1560

$\S 20$ Bußgeldvorschriften -1561

Schrifttum 1561

Alphabetisches Stichwortverzeichnis - 1562

A. Allgemeines - 1563

I. Entstehungsgeschichte -1563

II. Schutzzweck und Gesetzessystematik — 1564

III. Praktische Bedeutung - 1565

B. Objektiver Tatbestand - 1566

I. Unerlaubte Telefonwerbung (Abs. 1 Nr. 1) -1566

II. Verstöße gegen die Berichtspflicht (Abs. 1 Nr. 2) -1568

III. Verstöße gegen Pflichten aus einer Rechtsverordnung oder einer hierauf beruhenden vollziehbaren Anordnung (Abs. 1 Nr. 3) -1569

C. Subjektiver Tatbestand; Fahrlässigkeit - 1570

I. Vorsatz - 1571

II. Fahrlässigkeit 1572

D. Rechtswidrigkeit - 1572

E. Versuch, Vollendung und Beendigung - 1573

F. Konkurrenzen - 1573

G. Bußgeld und weitere Rechtsfolgen - 1574

H. Verjährung — 1576

I. Bußgeldverfahren -1576

§ 23 GeschGehG Verletzung von Geschäftsgeheimnissen — 1578

Schrifttum -1581

Zu § 23 GeschGehG und zur RL (EU) 2016/943 (GeschGehRL) - 1581

$\mathrm{Zu} \S \S 17$ bis 19 UWG a. F. - 1582

Alphabetisches Stichwortverzeichnis - 1587

A. Allgemeines -1588

I. Überblick zum GeschGehG 1588

II. Allgemeines zu § $23 \mathrm{GeschGehG} 1594$

III. Entstehungsgeschichte des § 23 GeschGehG 1597

B. Geschäftsgeheimnis - 1598

I. Allgemeines 1598

II. Definition des Betriebs- und Geschäftsgeheimnisses i. S.d § 17 UWG a. F. — 1598

III. Definition des Geschäftsgeheimnisses i. S.d § 23 GeschGehG

(§ 2 Nr. 1 GeschGehG) — 1599

IV. Beispiele für Geschäftsgeheimnisse - 1612

C. Betriebsspionage ( $§ 23$ Abs. 1 Nr. 1 GeschGehG) — 1613

I. Objektiver Tatbestand -1614

II. Subjektiver Tatbestand -1619 
D. Eigeneröffnete Geheimnishehlerei (§ 23 Abs. 1 Nr. 2 GeschGehG) -1622

I. Objektiver Tatbestand -1622

II. Subjektiver Tatbestand -1626

E. Geheimnisverrat durch Beschäftigte ( $§ 23$ Abs. 1 Nr. 3 GeschGehG) -1626

I. Objektiver Tatbestand - 1626

II. Subjektiver Tatbestand - 1631

F. Fremderöffnete Geheimnishehlerei ( $§ 23$ Abs. 2 GeschGehG) -1632

I. Objektiver Tatbestand -1632

II. Subjektiver Tatbestand - 1633

G. Vorlagenfreibeuterei (§ 23 Abs. 3 GeschGehG) -1634

I. Allgemeines -1634

II. Objektiver Tatbestand - 1635

III. Subjektiver Tatbestand - 1640

H. Qualifikationen ( $§ 23$ Abs. 4 GeschGehG) -1641

I. Allgemeines - 1641

II. Gewerbsmäßiges Handeln (§ 23 Abs. 4 Nr. 1 GeschGehG) - 1641

III. Wissen um Auslandsnutzung ( $\$ 23$ Abs. 4 Nr. 2 GeschGehG) -1642

IV. Eigennutzung im Ausland ( $\$ 23$ Abs. 4 Nr. 3 GeschGehG) -1642

I. Spezielle Erlaubnis- und Ausnahmeregelungen — 1643

I. Erlaubte Handlungen ( $\$ 3$ GeschGehG) -1643

II. Ausnahmen ( $§ 5$ GeschGehG) -1644

J. Rechtswidrigkeit -1654

I. Besondere Rechtfertigungsgründe -1655

II. Einwilligung - 1655

III. Anzeige-, Auskunfts- und Aussagepflichten - 1655

IV. Schuldrechtliche Auskunftsansprüche - 1656

V. Rechtfertigender Notstand (§ 34 StGB) -1656

VI. Mutmaßliche Einwilligung - 1657

VII. Rechtfertigende Pflichtenkollision - 1657

K. Schuld - 1657

L. Versuch (§ 23 Abs. 5 GeschGehG) - 1657

M. Beihilfehandlungen von Medienangehörigen (§ 23 Abs. 6 GeschGehG) -1658

N. Auslandstaten (§ 23 Abs. 7 S. 1 GeschGehG) -1659

I. Allgemeines 1659

II. Auslandstaten mit besonderem Inlandsbezug - 1660

III. Auslandstaten ohne besonderen Inlandsbezug - 1661

0. Versuch der Beteiligung und Rücktritt (§ 23 Abs. 7 S. 2 GeschGehG) -1661

I. Allgemeines 1661

II. Versuch der Beteiligung ( $\$ 23$ Abs. 7 S. 2 GeschGehG i. V. m. $\S 30$ StGB) -1663

III. Rücktritt vom Versuch (§ 23 Abs. 7 S. 2 GeschGehG i. V. m. § 31 StGB) -1665

P. Strafverfolgung ( $§ 23$ Abs. 8 GeschGehG) und Geheimhaltung, Privat- und Nebenklage $-1665$

I. Antragsdelikt ( $§ 23$ Abs. 8 Mod. 1 GeschGehG) -1665

II. Strafverfolgung von Amts wegen (§ 23 Abs. 8 Mod. 2 GeschGehG) -1667

III. Geheimhaltung — 1668

IV. Privat- und Nebenklage - 1669

V. Strafverfolgungsbehörden und Gerichte - 1669

Q. Konkurrenzen — 1669

R. Strafe und weitere Rechtsfolgen - 1671

S. Verjährung - 1672

Sachregister -1673 